\title{
Mixed adenoneuroendocrine carcinoma of the hepatic bile duct: a case report and review of the literature
}

\author{
Sulai Liu ${ }^{1 \dagger}$, Zhendong Zhong ${ }^{1,2 \dagger}$, Meng Xiao ${ }^{3}$, Yinghui Song ${ }^{1}$, Youye $\mathrm{Zhu}^{4}$, Bo Hu${ }^{1}$, Zengpeng Sun ${ }^{1}$, Weimin Yi ${ }^{1 *}$ \\ and Chuang Peng ${ }^{1^{*}}$
}

\begin{abstract}
Background: The World Health Organization's updated classification of digestive system neuroendocrine tumors in 2010 first proposed the classification of mixed adenoneuroendocrine carcinoma (MANEC). The incidence of biliary malignant tumors with neuroendocrine tumors accounts for less than $1 \%$ of all neuroendocrine tumors. Moreover, the incidence of hilar bile duct with MANEC is very rare.
\end{abstract}

Case presentation: A 65-year-old female patient came to our hospital for repeated abdominal pain for more than 4 months and skin sclera yellow staining for 1 week. Contrast-enhanced computed tomography imaging and magnetic resonance results suggested a hilar tumor for Bismuth-Corlette Type II. The patient underwent radical surgery for hilar cholangiocarcinoma. Finally, the patient was diagnosed with hilar bile duct MANEC, staged 1 (pT1NOMO) based on the eighth edition of the AJCC. Histopathology showed that the tumor was a biliary tumor with both adenocarcinoma and neuroendocrine carcinoma. No evidence of recurrence and metastasis after 20 months of follow-up.

Conclusions: We first reported a MANEC that originated in the hilar bile duct. As far as we known, there were few reports of biliary MANEC, and the overall prognosis was poor. We also found that the higher the Ki-67 index, the worse the prognosis of this type of patient. Radical surgery is the most effective treatment.

Keywords: Neuroendocrine tumor, Mixed adenoneuroendocrine carcinoma, Hepatic bile duct

\section{Background}

The most common pathological type of hilar cholangiocarcinoma is adenocarcinoma, accounting for more than 90\%. Others mainly include gland-squamous tumors, intraductal papillary tumors, mucinous gland tumors [1]. Mixed adenine neuroendocrine carcinoma (MANEC) is defined as a compound tumor of adenocarcinoma or squamous cell carcinoma mixed with neuroendocrine carcinoma (NEC) or neuroendocrine tumor (NET), with

\footnotetext{
*Correspondence: Ywm8012@sohu.com; pengchuangcn@163.com

†Sulai Liu and Zhendong Zhong have contributed equally to this work

${ }^{1}$ Department of Hepatobiliary Surgery, Hunan Provincial People's

Hospital/The First Affiliated Hospital of Hunan Normal University,

Changsha, Hunan Province, People's Republic of China

Full list of author information is available at the end of the article
}

each tumor accounting for at least $30 \%$ of the tumor. Meanwhile, these two histological components must be malignant [2]. Neuroendocrine neoplasms (NENs) are distributed in the neuroendocrine system, mainly from the neuroendocrine cells of the digestive system and respiratory tract. The diagnosis depends on histopathological examination [3].NENs can form a mixed carcinoma with malignant-tumors in the primary site, and neuroendocrine tumors of biliary mixed adenocarcinoma are rare in clinical practice [4].

Since the concept of MANEC was introduced in 2010, cases of MANEC have been continuously reported. Retrieving literature, there are only a few case reports describing the MANEC of the hepatic bile duct. However, there is no report of hepatic hilar bile duct MANEC. We

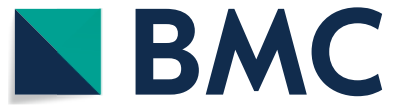

(c) The Author(s) 2020. This article is licensed under a Creative Commons Attribution 4.0 International License, which permits use, sharing, adaptation, distribution and reproduction in any medium or format, as long as you give appropriate credit to the original author(s) and the source, provide a link to the Creative Commons licence, and indicate if changes were made. The images or other third party material in this article are included in the article's Creative Commons licence, unless indicated otherwise in a credit line to the material. If material is not included in the article's Creative Commons licence and your intended use is not permitted by statutory regulation or exceeds the permitted use, you will need to obtain permission directly from the copyright holder. To view a copy of this licence, visit http://creativecommons.org/licenses/by/4.0/. The Creative Commons Public Domain Dedication waiver (http://creativecommons.org/publicdomain/zero/1.0/) applies to the data made available in this article, unless otherwise stated in a credit line to the data. 
presented a patient with hepatic hilar bile duct MANEC here, and combined with the literature to summarize the characteristics of this disease.

\section{Case presentation Case history}

A 65-year-old female patient came to our hospital on January 11th, 2018 for repeated pain in the upper abdomen for 4 months and skin sclera for 1 week. At the time of admission, the patient's skin and sclera turned yellow, mild abdominal pain, no fever and abdominal tenderness. Laboratory examination showed obstructive jaundice changes accompanied by an increase in Carbohydrate antigen 19-9 (CA19-9).

The patient underwent a contrast-enhanced computed tomography $(\mathrm{CT})$ and magnetic resonance imaging examination in our hospital. The results showed that soft tissue-like density lesions were seen in the bile duct of the hilar region, and the lesions involved the upper segment of the common bile duct. Enhanced scanning lesions showed mild enhancement. Considering Bismuth-Corlette II type of hilar cholangiocarcinoma, intrahepatic bile duct dilatation was evident above the tumor site. The structure of the portal hepatic hilum area was not clear, considering the possibility of tumor invasion. Multiple lymph nodes were swollen after the retroperitoneum. Magnetic Resonance Cholangiopancreatography (MRCP) showed hepatic hilar bile duct truncation, and the intrahepatic and extrahepatic bile ducts were significantly dilated above the obstruction. No significant expansion of the main pancreatic duct. No intrahepatic or distant metastases were found (Fig. 1).

After detailed preoperative discussion and preparation, resection of the extrahepatic bile ducts with concomitant radical lymphadenectomy and Roux-en-Y cholangiojejunostomy was performed. During the operation, hepatic cholestasis was observed, and the hilar bile duct had a mass of $2 \mathrm{~cm} \times 3 \mathrm{~cm} \times 2 \mathrm{~cm}$. The portal vein has not been invaded by the tumor. No perioperative complications occurred and the patient was discharged on the 12th day after surgery. According to the eighth edition of the AJCC tumor staging [5], this patient was stage 1 (pT1N0M0). After she discharged from hospital, she was followed-up by telephone once a month, and she came to hospital for liver function test, CA19-9 and abdomen contrast-enhanced computed tomography. Until now, no abdominal pain, no skin or scleral yellowing and other abnormalities, no signs of recurrence and metastasis.

\section{Histology and immunohistochemistry}

A $2 \times 1.8 \times 1 \mathrm{~cm}$ mass was seen in the hilar bile duct and invaded the entire bile duct wall. At light microscope, two tumor components of adenocarcinoma and neuroendocrine cancer could be seen as collision type. Adenocarcinoma cells were columnar, cubic, and nuclear division were rare. Neuroendocrine cancer tumor tissues were solid, flaky, trabecular or organ-like. The cells were small and round, and the cytoplasm were sparse. There were abundant sinusoids in the middle, and the Ki-67 index was $70 \%$. Neurological invasion was occurred, no intravascular tumor thrombus was seen, and no involvement of cancer in the bile duct margin. No lymph node metastasis.

Immunohistochemistry and special staining: CK7 cholangiocarcinoma $(+)$, CK19 (+), CD34 vascular $(+)$, NSE nerve $(+)$, Ki67 (+, 70\%), CgA, Syn neuroendocrine carcinoma $(+)$, p53 (cholangiocarcinoma $3+$, neuroendocrine carcinoma $2+$ ), VG (+) (Fig. 2).
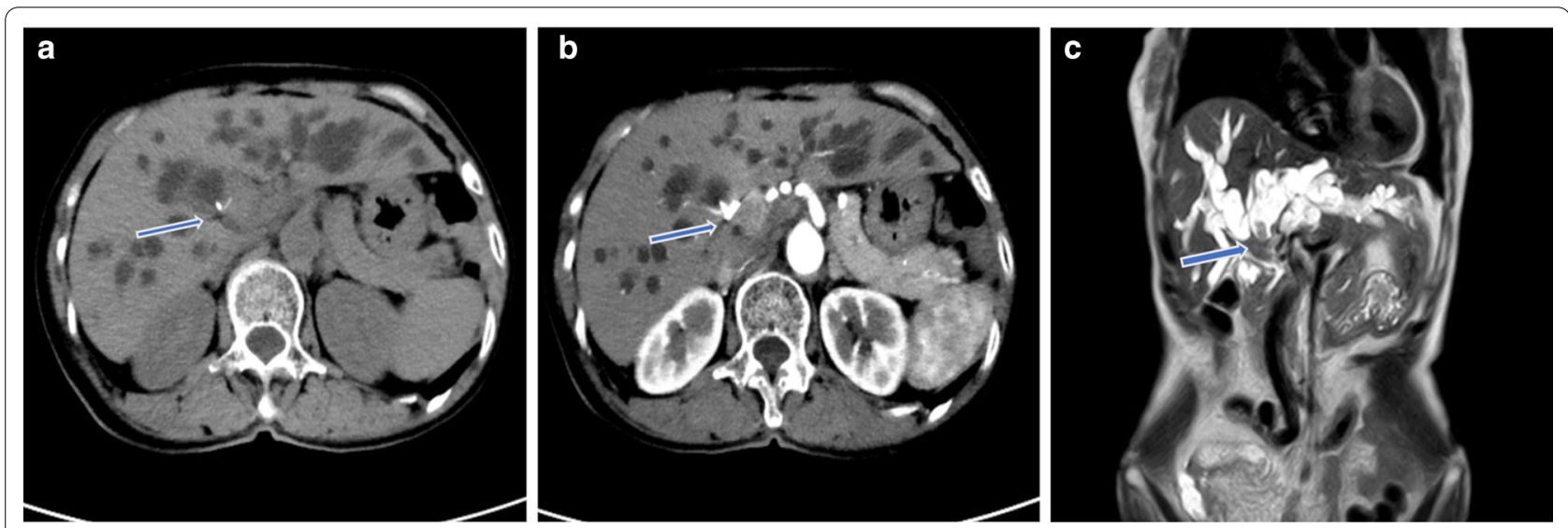

Fig. 1 Preoperative imaging findings showed hilar bile duct mass. a CT scan showed that the liver tissue area saw soft tissue-like density lesions, and the bile ducts above the lesions were significantly expanded. $\mathbf{b}$ CT enhanced scan indicates that the lesion is mildly enhanced. $\mathbf{c}$ MRCP showed truncation of the hilar bile duct, and the intrahepatic bile duct was significantly dilated above the obstruction 


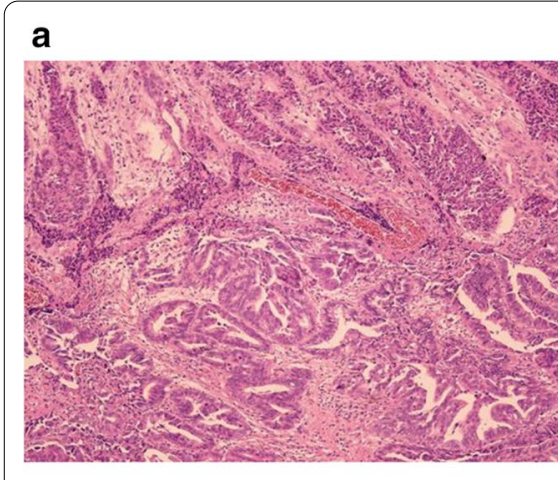

d
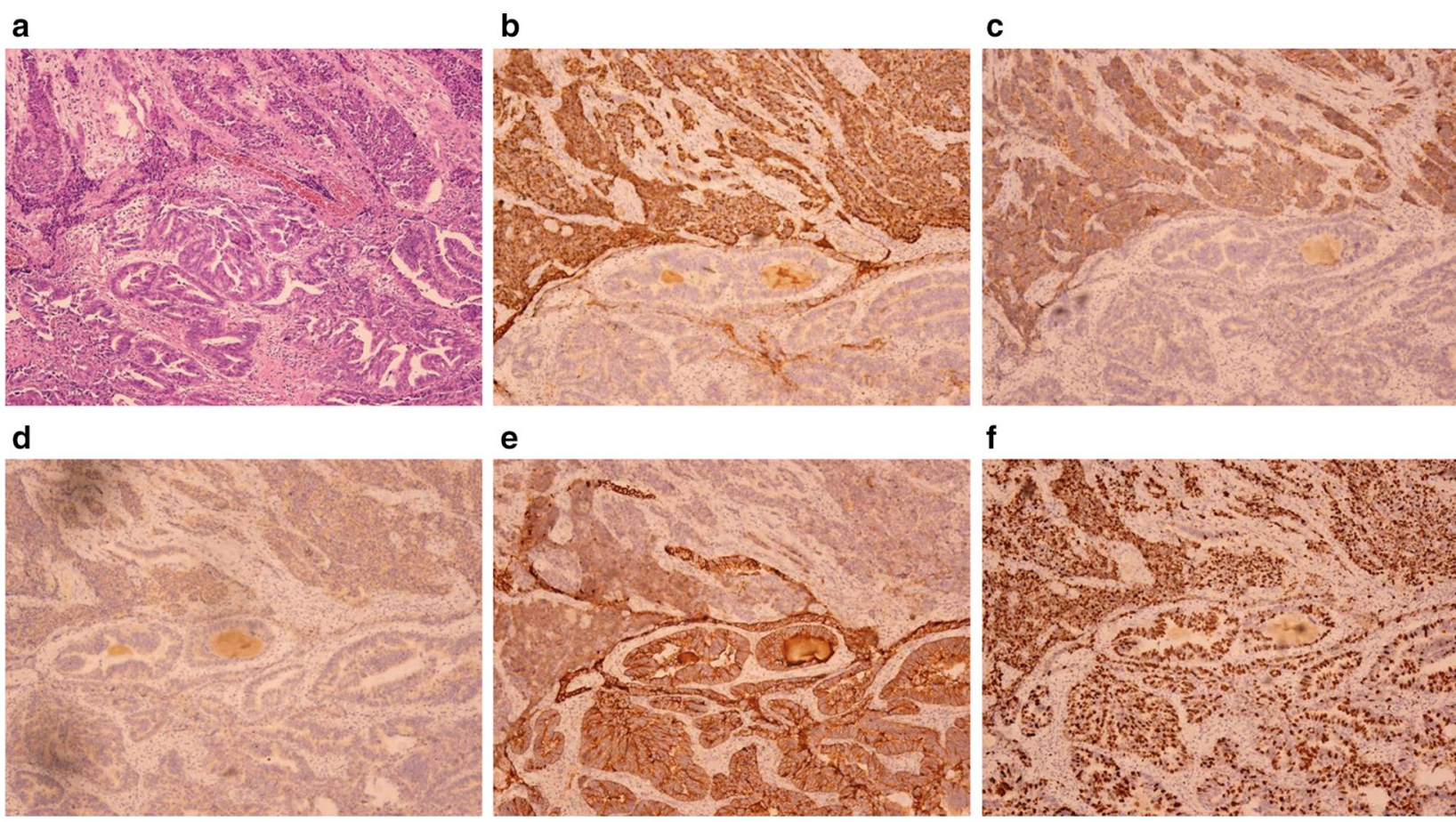

e

\section{$f$}
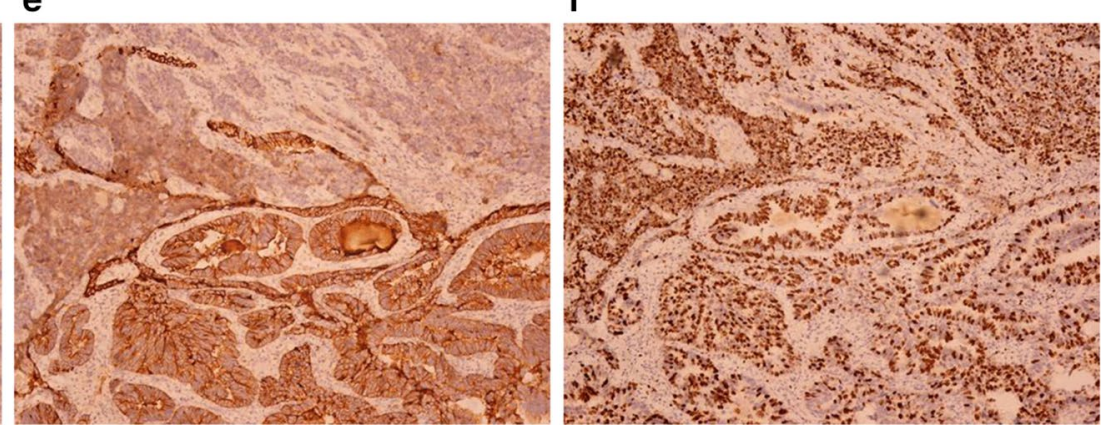

Fig. 2 Histology and immunohistochemistry. aNeuroendocrine carcinoma and tubular adenocarcinoma [hematoxylin and eosin staining (HE), $\times 100]$. b Cytoplasmic diffuse positive in CgA cells (DAB color development, $\times 200)$. Immunohistochemical staining. c Syncytoplasmic cytoplasmic positive, $\times 200$. $\mathbf{d}$ Neuronal specific enolase (NSE) positive in neuroendocrine tumor cells, $\times 100$. e Cytoplasmic cytokeratin 7 (CK7) diffuse positive in adenocarcinoma tumor cells, $\times 200$. f Cell proliferation index (Ki-67) cell nucleus positive, positive rate was 70\%, $\times 100$

\section{Discussion and conclusion}

$90 \%$ of the malignant tumors of the biliary system are adenocarcinomas, and other types of tumors are rare [6]. The proportion of biliary NENs to all NENs is less than $1 \%$. MANEC, which originated in the hilar bile duct, is rare. The World Health Organization's 2010 classification system classifies neuroendocrine tumors into neuroendocrine tumors (NET), neuroendocrine carcinomas (NEC), and MANEC. Subdivided into NET G1 phase: carcinoid, $<2$ mitotic cells per 10 high power fields, and/ or Ki-67 index $\leq 2 \%$. NET G2 phase: $2-20$ mitotic cells per 10 high power fields, and/or Ki-67 index between 3 and 20\%. NET G3: Neuroendocrine carcinoma with $>20$ mitotic cells per 10 high power fields, and/or Ki-67 index $>20 \%$, and MANEC $[7,8]$.

According to the location of tumor components, MANEC can be divided into three subtypes: composite neoplasms, collision neoplasms and combined neoplasms. Composite neoplasms refers to two different types of tumors separated from each other with normal tissue separation in between. Collision neoplasms are the neuroendocrine and exocrine components occur in separate areas of the same lesion with no normal tissue spacing in between. While in other MANECs they are intimately and diffusely admixed (combined neoplasms)
[9]. In this case, the patient's tumor was located in the hilar bile duct, and no intrahepatic bile duct was accumulated. The two tumor components were separated from each other, which was a collision type. The proportion of the two tumor components was greater than $30 \%$.

By searching the literature, we found that biliary MANEC is rare, and only one of the retrospective studies mentioned two cases of hepatic MANEC. But it lacked corresponding analysis [10] 0.10 cases of biliary MANEC patients were summarized [10-18]. Including 4 cases of distal bile duct, 2 cases of hilar bile duct, 1 case of common bile duct, 1 case of intrahepatic bile duct, 1 case of cystic duct, and 1 case of extrahepatic bile duct. The symptoms of this type of patients mainly include abdominal pain and yellowing of the skin or sclera. CT/ magnetic resonance imaging is consistent with the characteristics of biliary adenocarcinoma. Accurate diagnosis depends on histopathological examination. Preoperative tumor markers were generally not elevated. All patients underwent radical surgery. The type of tumor was mainly mixed with adenocarcinoma and neuroendocrine carcinoma. Only two cases were considered as collision type MANEC. At present, the pathogenesis of bile duct MANEC is unclear. Some scholars believe that it may be related to the neuroendocrine of a small amount of 
enterochromaffin cells distributed on the biliary system [19], and some are believed to be caused by congenital developmental abnormalities or long-term chronic inflammation. Regarding the pathogenesis of MANEC, Harada et al. [20] analyzed the histopathological features of 274 cases of biliary neuroendocrine tumors, suggesting that hepatic stem cells may be involved in the differentiation of neuroendocrine tumor cells. It is also suggested that bile duct stones combined with cholangitis is one of the important risk factors that may induce the accidental generation of neuroendocrine cells. Some scholars have also suggested that the Notch1-Hes1 signal axis inhibits the differentiation of neuroendocrine cells and maintains the tubular or acinar characteristics of adenocarcinoma and normal biliary cells. The interruption of this signal axis may be related to the occurrence of biliary MANEC [21].

All MANEC are basically diagnosed by histopathological examination [15]. It is reported that cytological biopsies by ERCP or endoscopic ultrasound-guided fine needle puncture etc. were used before surgery. But only $\mathrm{AC}$ or NET was detected, and the postoperative diagnosis was MANEC [22]. The possible reason may be the diverse components of MANEC, and the complicated structural distribution of AC and NEC leading to incomplete sampling. Therefore, it is still necessary to explore other effective examination methods to assist the preoperative medical examination to make a diagnosis of the bile duct MANEC. As a rich blood supply tumor, NET is obviously strengthened in the CT phase of the arterial phase, but the cholangiocarcinoma is poorly strengthened. It is reported that MANEC with obvious enhancement in the $\mathrm{CT}$ arterial phase, which proves the existence of the NET component and assists before surgery, which supported a new direction for the diagnosis of bile ducts MANEC [15]. Preoperative lymph node biopsy can also be used as an auxiliary diagnostic method. When the metastatic component is NETG2 or NEC, the possibility of MANEC should be considered [10].

Because the MANEC of the biliary tract is rare, the clinical research lacks sufficient data, and the existing case reports indicate that the prognosis is poor. There is literature analysis that the tumor-free survival and overall survival of the biliary neuroendocrine tumor stage are 5.8 (range 0.4-53.6) months, and 13.7 (range 0.7-102.1) months, respectively. And the prognosis of NET is significantly better than NEC and MANEC. The higher the Ki67 index, the easier the tumor recurs and the poor prognosis [23]. It has been reported in the literature that adjuvant radiotherapy and chemotherapy after neuroendocrine tumors may improve the prognosis, but radical surgical resection is still the most effective treatment [10, 21]. This patient also underwent radical surgery. For hilar cholangiocarcinoma treatment, our central has extensive treatment experience [24]. She still survived very well after surgery and did not receive any radiotherapy or chemotherapy and other adjuvant treatment.

At present, most of the evidence indicates that the treatment of biliary tract MANEC should be based on the grade of adenocarcinoma or NET, and the high grade tumor as a reference for postoperative adjuvant therapy. Multiple retrospective analyses have pointed out that the $\mathrm{Ki}-67$ index is an independent risk factor for predicting the prognosis of neuroendocrine carcinoma. When the $\mathrm{Ki}-67$ index is higher than $55 \%$, it is highly prognostic [8, $25,26]$.

In conclusion, the overall survival of biliary MANEC is still lacking in large sample statistics. We reported the first case of MANEC in the hilar bile duct. It is hoped that reference materials will be provided for the diagnosis, treatment and prognosis analysis of such patients.

\section{Abbreviations \\ ADC: Adenocarcinoma; CA19-9: Carbohydrate antigen 19-9; CBD: Common bile duct; CK7: Cytoplasmic cytokeratin 7; CT: Computed tomography; HE: Hematoxylin and eosin staining; IPNB: Intraductal papillary neoplasm of the bile duct; MANEC: Mixed adenoneuroendocrine carcinoma; MRCP: Magnetic resonance cholangiopancreatography; NEC: Neuroendocrine carcinoma; NENs: Neuroendocrine neoplasms; NET: Neuroendocrine tumor; NSE: Neuronal specific enolase; PD: Pancreaticoduodenectomy; UICC: Union for international cancer control.}

\section{Acknowledgements}

Not applicable.

\section{Authors' contributions}

ZZD and XM wrote the manuscript. HB and SZP participated in the collection and follow-up of data. PC, YWM and LSL assisted in the preparation of the manuscript and critically reviewed the manuscript. ZYY contributed to the pathological diagnosis of this patient. SYH revised the manuscript. All authors read and approved the final manuscript.

\section{Funding}

This work was financially supported by following funds (founder is Dr Sulai Liu): National science founding of china (81902017)/Hunan Provincial Natural Science Foundation of China (Grant No. 2019JJ50320)/ Central Guidance of Local Science and Technology Development Fund (Grant No. 2018CT5008)/ Project of Scientific Research of Traditional Chinese Medicine in Hunan (Grant No. 201809)

\section{Availability of data and materials}

Data and materials are included in the manuscript.

\section{Ethics approval and consent to participate}

Written informed consent was obtained from the patient to participate to this case report.

\section{Consent to publish}

All authors agree to the publication of this article. Written informed consent was obtained from the patient for publication of this case report and any accompanying images. A copy of the written consent is available for review by the Editor of this journal.

\section{Competing interests}

The authors declare that they have no financial or commercial conflict of interest. 


\begin{abstract}
Author details
1 Department of Hepatobiliary Surgery, Hunan Provincial People's Hospital/ The First Affiliated Hospital of Hunan Normal University, Changsha, Hunan Province, People's Republic of China. ${ }^{2}$ Department of Hepatobiliary Surgery, Changsha County People's Hospital/Hunan Provincial People's Hospital Xingsha Campus, Changsha, China. ${ }^{3}$ Department of Medical Center, Hunan Provincial Hospital for Occupational Diseases, Changsha, China. ${ }^{4}$ Department of Pathology, Changsha County People's Hospital/Hunan Provincial People's Hospital Xingsha Campus, Changsha, China.
\end{abstract}

Received: 11 December 2019 Accepted: 19 November 2020

Published online: 25 November 2020

\section{References}

1. Gershenwald JE, Scolyer RA, Hess KR, et al. Melanoma staging: evidencebased changes in the American Joint Committee on Cancer eighth edition cancer staging manual. CA Cancer J Clin. 2017;67(6):472-92.

2. Rindi G, Petrone G, Inzani F. The 2010 WHO classification of digestive neuroendocrine neoplasms: a critical appraisal four years after its introduction. Endocr Pathol. 2014;25(2):186-92.

3. Yao JC, Hassan M, Phan A, et al. One hundred years after "carcinoid": epidemiology of and prognostic factors for neuroendocrine tumors in 35,825 cases in the United States. J Clin Oncol. 2008;26(18):3063-72.

4. Kim JY, Hong SM. Recent updates on neuroendocrine tumors from the gastrointestinal and pancreatobiliary tracts. Arch Pathol Lab Med. 2016;140(5):437-48.

5. Chun YS, Pawlik TM, Vauthey JN. 8th edition of the AJCC cancer staging manual: pancreas and hepatobiliary cancers. Ann Surg Oncol. 2018;25(4):845-7.

6. Zheng Z, Chen C, Li B, et al. Biliary neuroendocrine neoplasms: clinical profiles, management, and analysis of prognostic factors. Front Oncol. 2019;9:38.

7. Fléjou JF. WHO Classification of digestive tumors: the fourth edition. Ann Pathol. 2011;31(5 Suppl):S27-31.

8. Delektorskaia WV, Kushliskiĭ NE. Neuroendocrine tumors of digestive system: morphologic spectrum and cell proliferation (Ki67 index). Vestn Ross Akad Med Nauk. 2013;5:28-37.

9. La Rosa S, Marando A, Sessa F, et al. Mixed adenoneuroendocrine carcinomas (MANECs) of the gastrointestinal tract: an update. Cancers (Basel). 2012;4(1):11-30.

10. Harada K, Sato Y, Ikeda H, et al. Clinicopathologic study of mixed adenoneuroendocrine carcinomas of hepatobiliary organs. Virchows Arch. 2012;460(3):281-9.

11. Onishi I, Kitagawa H, Harada K, et al. Intraductal papillary neoplasm of the bile duct accompanying biliary mixed adenoneuroendocrine carcinoma. World J Gastroenterol. 2013;19(20):3161-4.

12. Lee SW, Lee IS, Cho YK, et al. A case of mixed adenoneuroendocrine carcinoma of the common bile duct: initially diagnosed as cholangiocarcinoma. Korean J Pathol. 2014;48(6):445-8.
13. Zheng SL, Yip VS, Pedica F, et al. Intrahepatic bile duct mixed adenoneuroendocrine carcinoma: a case report and review of the literature. Diagn Pathol. 2015:10:204.

14. Takemoto YK, Abe T, Amano H, et al. Mixed adenoneuroendocrine carcinoma derived from the cystic duct: a case report. Int J Surg Case Rep. 2017;39:29-33

15. Izumo W, Higuchi R, Yazawa T, et al. A long-term recurrence-free survival of a patient with the mixed adeno-neuroendocrine bile duct carcinoma: a case report and review of the literature. Int J Surg Case Rep. 2017;39:43-50

16. Komo T, Kohashi T, Nakashima A, et al. Mixed adenoneuroendocrine carcinoma of the distal bile duct: a case report. Int J Surg Case Rep. 2017:39:203-7.

17. Siddiqui I, Kirks RC, Sastry AV, et al. Mixed adenoneuroendocrine carcinoma (MANEC) of the distal common bile duct arising from a choledochal cyst. Am Surg. 2018;84(2):319-21.

18. Zhang L, Yang Z, Chen $Q$, et al. Mixed adenoendocrine carcinoma in the extrahepatic biliary tract: a case report and literature review. Oncol Lett. 2019;18(2):1585-96

19. Kurumaya H, Ohta G, Nakanuma Y. Endocrine cells in the intrahepatic biliary tree in normal livers and hepatolithiasis. Arch Pathol Lab Med. 1989;113(2):143-7.

20. Uppin MS, Uppin SG, Sunil CS, Hui M, Paul TR, Bheerappa N. Clinicopathologic study of neuroendocrine tumors of gastroenteropancreatic tract: a single institutional experience. J Gastrointest Oncol. 2017;8(1):139-47.

21. Harada K, Sato Y, Ikeda H, Hsu M, Igarashi S, Nakanuma Y. Notch1-Hes1 signalling axis in the tumourigenesis of biliary neuroendocrine tumours. J Clin Pathol. 2013;66(5):386-91.

22. Song W, Chen W, Zhang S, Peng J, He Y. Successful treatment of gallbladder mixed adenoneuroendocrine carcinoma with neo-adjuvant chemotherapy. Diagn Pathol. 2012;7:163.

23. Kim J, Lee WJ, Lee $\mathrm{SH}$, et al. Clinical features of 20 patients with curatively resected biliary neuroendocrine tumours. Dig Liver Dis. 2011;43(12):965-70

24. Liu S, Liu X, Li X, et al. Application of laparoscopic radical resection for type III and IV hilar cholangiocarcinoma treatment. Gastroenterol Res Pract. 2020;2020:1506275

25. Milione M, Maisonneuve P, Pellegrinelli A, et al. Ki67 proliferative index of the neuroendocrine component drives MANEC prognosis. Endocr Relat Cancer. 2018;25(5):583-93.

26. Xie JW, Lu J, Wang JB, et al. Prognostic factors for survival after curative resection of gastric mixed adenoneuroendocrine carcinoma: a series of 80 patients. BMC Cancer. 2018;18(1):1021.

\section{Publisher's Note}

Springer Nature remains neutral with regard to jurisdictional claims in published maps and institutional affiliations.
Ready to submit your research? Choose BMC and benefit from:

- fast, convenient online submission

- thorough peer review by experienced researchers in your field

- rapid publication on acceptance

- support for research data, including large and complex data types

- gold Open Access which fosters wider collaboration and increased citations

- maximum visibility for your research: over 100M website views per year

At $\mathrm{BMC}$, research is always in progress.

Learn more biomedcentral.com/submissions 